\title{
Development of a Color Object Classification and Measurement System Using Machine Vision
}

\author{
Ngoc-Vu Ngo, ${ }^{1}$ Glen Andrew Porter, ${ }^{2}$ and Quang-Cherng $\mathrm{Hsu}^{3 *}$ \\ ${ }^{1}$ Division of Mechatronics, Department of Mechanical Engineering, Thai Nguyen University of Technology \\ 666, 3/2 Street, Thai Nguyen City 250000, Vietnam \\ ${ }^{2}$ Department of Electronic Engineering, National Kaohsiung University of Science and Technology \\ 415 Chien Kung Road, Sanmin District, Kaohsiung 80778, Taiwan, ROC \\ ${ }^{3}$ Department of Mechanical Engineering, National Kaohsiung University of Science and Technology, \\ 415 Chien Kung Road, Sanmin District, Kaohsiung 80778, Taiwan, ROC
}

(Received April 24, 2019; accepted July 31, 2019)

Keywords: machine vision, classification, color object, robot arm

Machine-vision-based reading and sorting devices have been used to measure and classify items. Here, we extend their application to the sorting and assembling of items identified by their geometry and color. In this study, we developed an improved machine vision system that is capable of discerning and categorizing items of distinct geometries and colors and utilizes a computer-controlled robotic system to manipulate and segregate these items. Hence, a machine vision system for an automatic classification process while operating a robotic arm is hereby developed. To obtain positioning information, the proposed system uses cameras that were mounted above the working platform to acquire images. Perspective and quadratic transformations were used to transform the image coordinates of the calibration system to the world coordinates by using a calibration procedure. By these methods, the proposed system can ascertain the two- and three-dimensional coordinates of the objects and automatically perform classification and assembly operations using the data collected from the visual recognition system.

\section{Introduction}

With the rapidly growing demand for industrial automation, flexible automatic assembly systems are one of the most useful tools for automated manufacturing processes. These systems are expected to integrate machine vision systems for various applications within automation systems and production lines.

Nowadays, machine vision has been widely applied in manufacturing technology for the inspection and defect detection of components and surfaces. For instance, Kavitha and Ashok applied machine vision to measuring the radial error of a lathe spindle while turning a master cylinder. $^{(1)}$ This system used a CMOS camera and a personal computer (PC). An image processing system was used to obtain variations of the center position of a master cylinder

*Corresponding author: e-mail: hsuqc@nkust.edu.tw https://doi.org/10.18494/SAM.2019.2412 
during spindle rotation. The radial error values of the spindle were evaluated by the Fourier series analysis of the center position of the master cylinder. Dworkin and Nye proposed a machine vision algorithm to measure hot-formed parts. ${ }^{(2)}$ In this study, the authors used CCD cameras to obtain desired images with visible light. Then, thresholding operations were conducted. Finally, the images were tested and processed. Di Leo et al. used a machine vision apparatus to design a measurement system for the online inspection of electromechanical parts. ${ }^{(3)}$ This system used two cameras for obtaining images of the parts to be measured. The measurement system was demonstrated to be efficient and to fully meet the specifications of the industrial-quality production control system. Shuxia et al. presented a machine vision measurement method involving cutting device diameter and the maximum rotating diameter of a mini-milling machine. ${ }^{(4)}$ In this study, a subpixel threshold segmentation algorithm was used to detect the edges of the mini-milling cutter. These two features were robustly and accurately determined through a successful application of the Hough transform and a linear regression. Their proposed machine vision system allowed a fast and accurate assessment of the cutter head dimensions. In a study by Ngo et al., a machine vision system using a double CMOS camera was built to measure the three-dimensional sizes of mechanical parts. ${ }^{(5)}$ The image data acquired from the double CMOS camera were transmitted to a computer for the calculation of parameters. With this proposed system, the sizes of parts could be determined rapidly and accurately. Xiang et al. proposed a measurement system using a two-camera machine vision setup to perform high-accuracy measurement of large automobile brake pad bayonets. ${ }^{(6)}$ After the edges of the linings were detected, the sizes were determined on the basis of a relative measurement principle by using a measurement equation and a linear fitting. The experimental results showed that the repeatable precision was $\pm 0.019 \mathrm{~mm}$, the average of biases was $0.003 \mathrm{~mm}$, and the average measurement time was an impressive $0.396 \mathrm{~s}$ per brake pad.

Among these applications, generalized procedures for adapting cameras to robot control applications using machine vision have been developed by several researchers. Blasco et al. used two vision systems mounted on an organic-produce-sorting machine. ${ }^{(7)}$ One of them was used to detect weeds and pass their coordinates to the controller of a robotic arm, and the other for correcting inertial perturbations induced in the position of the end effector. The primary vision system was demonstrated to be capable of properly locating $84 \%$ of weeds and $99 \%$ of lettuce heads. Nerakae et al. presented an automatic assembly system comprising a selective compliance assembly robot arm (SCARA) using machine vision. ${ }^{(8)}$ They designed the primary prototype of the flexible automatic assembly system to pick and place target objects. Iscimen et al. presented a smart robotic arm motion using computer vision to identify objects from images automatically and perform the given tasks. ${ }^{(9)}$ Of special interest, an artificial neural network was used for object recognition, and an overall recognition accuracy of $98.30 \%$ was achieved. Di Fulvio et al. proposed a stereoscopic vision system for conducting dimensional measurements in an industrial robotics application. ${ }^{(10)}$ In their research, a camera mounted on a six-axis robotic manipulator can calculate the world coordinates of target objects. Experimental results have shown that the measurement accuracy was strongly affected by the calibration accuracy. In addition, Rai et al. proposed a computer vision system for the detection and autonomous manipulation of objects on a target surface. ${ }^{(1)}$ Through an iterative training 
algorithm, a robotic arm with six degrees of freedom (DOF) can pick up objects and place them toward a destination. The system applied a center-of-mass-based computation, using a filtering and color segmentation algorithm, to locate both the target and position of the robotic arm. In another research, Tsarouchi et al. used a vision system for the robotic handling of randomly placed objects. ${ }^{(12)}$ They developed a method of calculating the coordinates of a part's features in the world coordinate system (WCS) using images captured by a camera. Such a method was implemented within a software tool using MATLAB and tested on the feeding of shaver handles into industrial manufacturing equipment.

In machine vision technology, the performance of a vision system largely depends on the calibration accuracy. You and Zheng presented an accurate and practical calibration method for a roadside camera using two vanishing points. ${ }^{(13)}$ To improve the accuracy of calibration results, they employed multiple observations of vanishing points and proposed a dynamic calibration method to correct the camera's parameters. The proposed method is suitable for outdoors, i.e., traffic scenarios. Shin and Mun proposed a new calibration method for a multicamera setup by a wand dance procedure, ${ }^{(14)}$ by which the calibration parameters were first estimated by the direct linear transformation (DLT) method. Then, the parameters estimated in the first step were improved iteratively through a nonlinear optimization by the wand dance procedure. Experimental results showed that the proposed wand method holds great promise for increasing the overall accuracy of the DLT algorithm and provides better user convenience. Deng et al. proposed a relationship model for camera calibration in which the geometric parameters and the lens distortion effect of the camera were taken into account to unify the WCS, the camera coordinate system (CCS), and the image coordinate system (ICS). ${ }^{(15)}$ In their study, the proposed algorithm can avoid local optimums and complete the visual identification tasks accurately. Ji et al. presented a novel design for an automatic and complete parameter calibration system. ${ }^{(16)}$ By using information from camera sensor networks to construct a $3 \mathrm{D}$ texture map, they developed an intelligent space data-acquisition method. The designed largescale scanning technique using a laser scanner was investigated by Wang et al., who performed both point and surface measurements to minimize errors, and the obtained values were used to calculate the uncertainty for the measurements. ${ }^{(17)}$ The measurement deviation and statistical values of variance were determined by Palousek et al. for the 3D digitization of glossy surfaces coated with a titanium oxide coating or a chalk powder spray. ${ }^{(18)}$ A 3D optical scanner, ATOS III Triple Scan, was used for capturing and evaluating the data, which revealed a significant difference between the chalk and the titanium matte coatings.

In this study, a machine vision system was used in conjunction with a robotic arm with six DOF for the classification and assembly of color geometrical solids into a module. This system included three high-resolution CMOS cameras and a PC. Here, a double CMOS camera was used to capture the images of assembly parts and modules. The assembly parts consisted of different geometric solids, including cubes, spheres, triangular prisms, and hexagonal prisms. The modules contained holes of different profile geometries, such as triangular, rectangular, and circular holes. Before capturing the images, all three cameras had to be calibrated to increase their accuracies. In the calibration work, the generalized perspective transformation and the quadratic transformation were used to determine the relationship between the ICS and the WCS. 
Then, the images of the color objects were processed using image processing software. After obtaining the desired images, the assembly parts were accurately recognized within the models by Blob analysis. The proposed system can recognize any general 2D and 3D color objects. Both the accuracy and success rate of the proposed system were nearly $100 \%$.

The remainder of this paper is organized as follows. In Sect. 2, we present the materials and the experimental setup, including a description of the proposed model, the machine vision system, and the robotic workstation platform. The implementation of the proposed technique is introduced in Sect. 3, as are the experimental procedures. Here, we describe the research methodology, including coordinate calibration methods, the calculations of the web-cam resolution, and how the orientations of triangular holes (target objects) were determined. The results and discussion, in which we evaluate the comprehensive performance of our proposed method, will be covered in Sects. 4 and 5, respectively. In Sect. 6, we conclude this work.

\section{Materials and Experimental Setup}

\subsection{Platform description}

Figure 1 shows the components of the vision system. The overall hardware included a robotic arm (HIWIN), three CMOS cameras, and a PC. The double CMOS camera C615 and the third camera C525 of the proposed system were mounted on a lattice above the platform. The double camera was used to capture the assembly modules, and the C525 camera was used to capture the assembly parts. The 3D-image-based vision equipment required an intelligent image analysis software program and an object detection algorithm. The proposed framework combined the control and image processing to perform the desired operations.

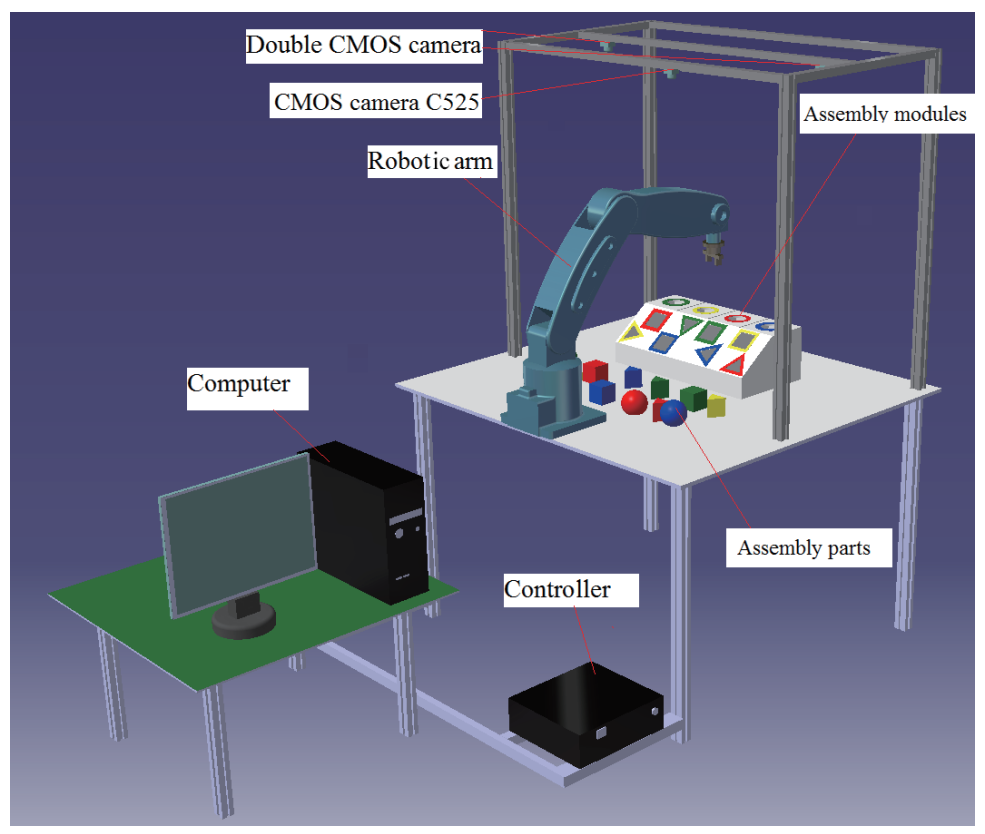

Fig. 1. (Color online) Experimental system. 
In a typical application, once a user's command was encountered, the workspace was scanned, and the corresponding images were captured. These images were processed to identify the target objects and make a center marker for each object. The acquired coordinates were then sent to the robotic arm to reach toward the target position. The various assembly parts, randomly placed within the workspace, included a sphere, a cube, a triangular prism, and a hexagonal prism, each of which was colored red, yellow, green, or blue. The assembly modules included four boxes for the classification process. Each box possessed circular, square, or triangular holes corresponding to the permutations of the first three aforementioned solid geometries and four colors. The circular hole was intended to accommodate the spheres and hexagonal prisms.

\subsection{Flowchart progression of the proposed system}

As shown in Fig. 2, once the proposed system received a command from the user, the camera calibration processes, including 2D and 3D calibrations, were implemented first, and then the workspace was scanned to acquire the desired images. The acquired images were processed to classify the color, and then Blob analysis was used to calculate the properties of blobs, such as the area, perimeter, and compactness, to identify the target object, and formulate a marker for their centers. Afterwards, the measurement data were sent to the robotic arm for manipulation.

\section{Research Methodology}

\subsection{Quadratic transformation}

With the 2D coordinate calibration, we used a quadratic transformation in this study using a regression analysis to extract the point coordinates for the calibration process. In this work, lens distortion was not considered. However, it can be used to predict the positions of points

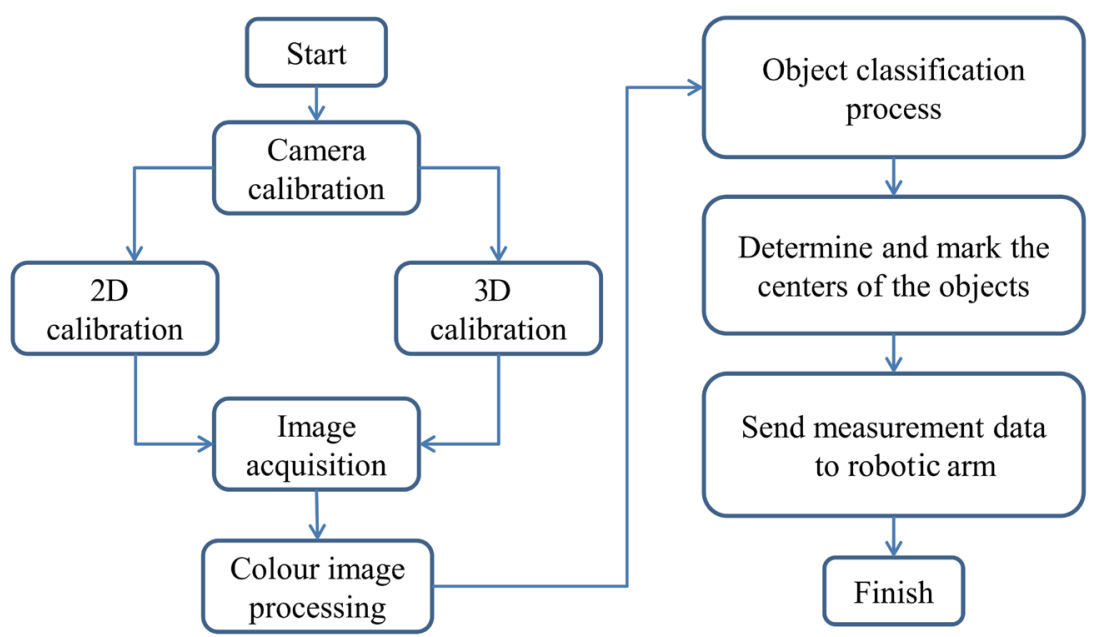

Fig. 2. Flowchart schema of the proposed system. 
in the WCS from the information provided by images. ${ }^{(19)}$ By this method, we found that the relationship between the image coordinates of point $I(x, y)$ and the world coordinates of point $W(X, Y)$ obey the following equation:

$$
\left\{\begin{array}{l}
X=a_{1} x^{2}+b_{1} x y+c_{1} y^{2}+e_{1} x+f_{1} y+g_{1} \\
Y=a_{2} x^{2}+b_{2} x y+c_{2} y^{2}+e_{2} x+f_{2} y+g_{2}
\end{array},\right.
$$

where $a_{1}, b_{1}, c_{1}, e_{1}, f_{1}$, and $g_{1}$, and $a_{2}, b_{2}, c_{2}, e_{2}, f_{2}$, and $g_{2}$ are the transformation coefficients. To find the two unknown variables in Eq. (1), $(X, Y)$, the transformation coefficients should be determined by regression analysis. ${ }^{(20)}$

\subsection{Perspective transformation}

With the $3 \mathrm{D}$ coordinate calibration, the perspective transformation was used to calibrate the coordinates. As shown in Fig. 3, an arrangement was selected such that the origin is at the center of the image, and the lens center was at coordinates $(0,0, \lambda)$, where $\lambda$ is the focal length of the lens. Figure 4 shows a generalized perspective projection of a 3D point in the WCS $(X, Y, Z)$, named $\boldsymbol{w}_{\boldsymbol{h}}$, onto an image plane with coordinates $(x, y, z)$, named $\boldsymbol{c}_{\boldsymbol{h}}$. In this figure, the camera was mounted at $G_{0}\left(X_{0}, Y_{0}, Z_{0}\right)$. The center of the image plane deviates from $G_{0}$ and is denoted by the vector $\boldsymbol{r}^{(5)}$

A generalized perspective transformation can be expressed as Eq. (2), which involves the transformation of the two coordinate systems.

$$
c_{h}=A C R G w_{h},
$$

where $\boldsymbol{C}$ and $\boldsymbol{G}$ are translation matrices, $\boldsymbol{R}$ is the rotation matrix, and $\boldsymbol{A}$ is a specific perspective transformation matrix.

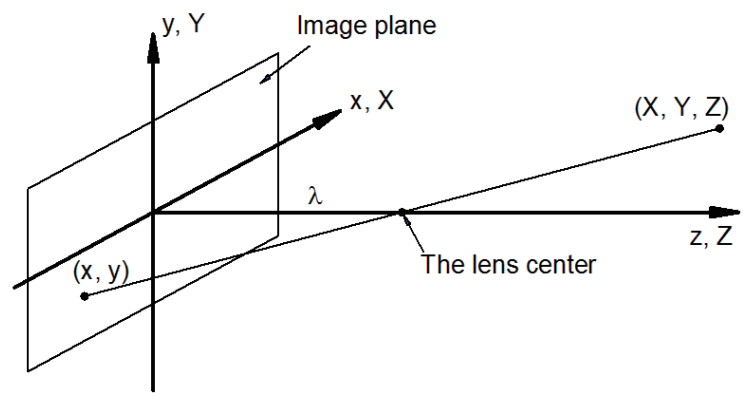

Fig. 3. Specific perspective projection model. ${ }^{(5)}$

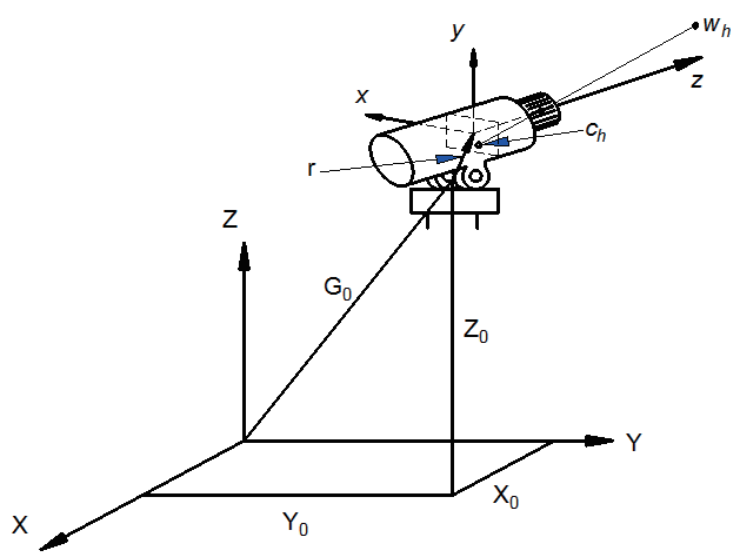

Fig. 4. (Color online) Generalized perspective projection model. $^{(5)}$ 
The product of $A C R G$ is recruited as $\boldsymbol{P}=\boldsymbol{A C R} \boldsymbol{G}$. A homogeneous $4 \times 4$ matrix representation for the $\boldsymbol{P}$ matrix can thus be described. Therefore, the generalized perspective transformation can be expressed by Eq. (3), where $s$ is the scale factor.

$$
\left[\begin{array}{c}
s x \\
s y \\
s z \\
s
\end{array}\right]=\left[\begin{array}{llll}
p_{11} & p_{12} & p_{13} & p_{14} \\
p_{21} & p_{22} & p_{23} & p_{24} \\
p_{31} & p_{32} & p_{33} & p_{34} \\
p_{41} & p_{42} & p_{43} & p_{44}
\end{array}\right]\left[\begin{array}{c}
X \\
Y \\
Z \\
1
\end{array}\right]
$$

From Eq. (3), image coordinates $(x, y)$ can be calculated using Eq. (4).

$$
\left\{\begin{array}{l}
x=\frac{s x}{s}=\frac{p_{11} X+p_{12} Y+p_{13} Z+p_{14}}{p_{41} X+p_{42} Y+p_{43} Z+p_{44}} \\
y=\frac{s y}{s}=\frac{p_{21} X+p_{22} Y+p_{23} Z+p_{24}}{p_{41} X+p_{42} Y+p_{43} Z+p_{44}}
\end{array}\right.
$$

In Eq. (3), the $\boldsymbol{P}$ matrix includes 16 variables, but four of these variables $\left(p_{31}, p_{32}, p_{33}, p_{34}\right)$ are not necessary in the calculation process. Thus, only 12 variables remain to be found, which are $p_{i j}(i=1,2,4 ; j=1, \ldots, 4)$. To find the 12 variables of the $\boldsymbol{P}$ matrix, six calibration points in the WCS were used; these points correspond to the image points in each image plane. After the 12 variables of the $\boldsymbol{P}$ matrix were found, using the iteration $p_{i j}(i=1,2,4 ; j=1, \ldots, 4)$, they were substituted into Eq. (4) to reconstruct the world coordinates. As can be seen, there are three unknown variables $(X, Y, Z)$ in Eq. (4).

To solve Eq. (4), a double camera was used to obtain two image planes, which were the left and right image planes. ${ }^{(5)}$ Each of these two image planes provides six points on the sample, which correspond to each other. In Eq. (5), the superscript (1) represents the left image plane created by the left camera, and the superscript (2) represents the right image plane created by the right camera.

$$
\left[\begin{array}{lll}
p_{11}^{(1)}-x^{(1)} p_{41}^{(1)} & p_{12}^{(1)}-x^{(1)} p_{42}^{(1)} & p_{13}^{(1)}-x^{(1)} p_{43}^{(1)} \\
p_{21}^{(1)}-y^{(1)} p_{41}^{(1)} & p_{22}^{(1)}-y^{(1)} p_{42}^{(1)} & p_{23}^{(1)}-y^{(1)} p_{43}^{(1)} \\
p_{11}^{(1)}-x^{(2)} p_{41}^{(1)} & p_{12}^{(1)}-x^{(2)} p_{42}^{(1)} & p_{13}^{(1)}-x^{(2)} p_{43}^{(1)} \\
p_{21}^{(1)}-y^{(2)} p_{41}^{(1)} & p_{22}^{(1)}-y^{(2)} p_{42}^{(1)} & p_{23}^{(1)}-y^{(2)} p_{43}^{(1)}
\end{array}\right]\left[\begin{array}{c}
X \\
Y \\
Z
\end{array}\right]=\left[\begin{array}{l}
-\left(p_{14}^{(1)}-x^{(1)} p_{44}^{(1)}\right) \\
-\left(p_{24}^{(1)}-y^{(1)} p_{44}^{(1)}\right) \\
-\left(p_{14}^{(1)}-x^{(2)} p_{44}^{(1)}\right) \\
-\left(p_{24}^{(1)}-y^{(2)} p_{44}^{(1)}\right)
\end{array}\right]
$$

\subsection{Determination of orientation of triangular holes}

In the classification process, all the assembly parts should fit within the assembly modules with respect to their shape and color. The orientations of the spheres and cubes were not distinct from the holes' direction, so there was no need to identify their angle. However, 
the triangular prisms had a particular orientation that fit within the respective holes, so the orientations of these parts and triangular holes should be determined. To detect the orientation of the triangular holes in this system, the pixels were scanned from left to right and from top to bottom of the binary images. The first contact point between the scan line and the triangle was identified as the first point. Scanning was performed on both the left and right image planes. An illustration of this technique for finding the first points of all the triangular holes is shown in Fig. 5.

Using this scanning technique, we found that the triangular holes have, at most, four orientations, as shown in Figs. 6(a)-6(d). Figures 7(a) and 7(b) show images of the triangular

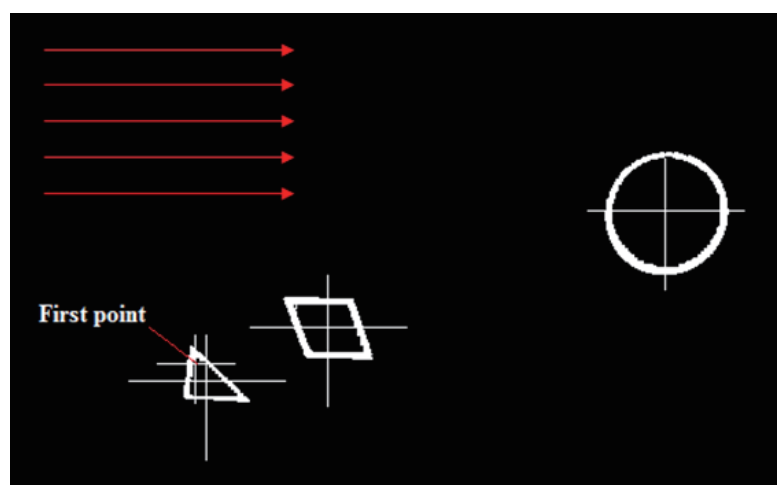

Fig. 5. (Color online) Scanning lines to find the first point of a triangular hole.

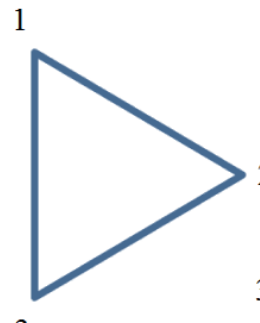

(a)

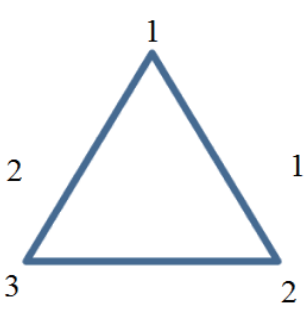

(b)

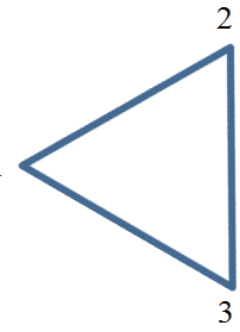

(c)

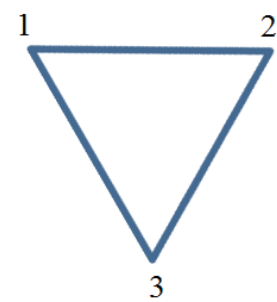

(d)

Fig. 6. Orientations of triangular holes. The vertices are numbered to identify the orientation of the triangle.

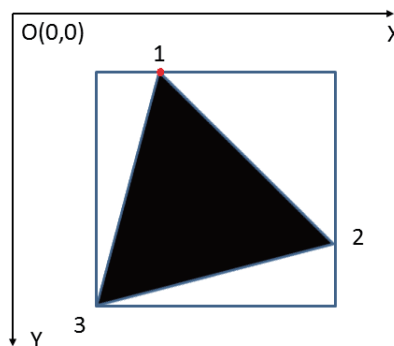

(a)

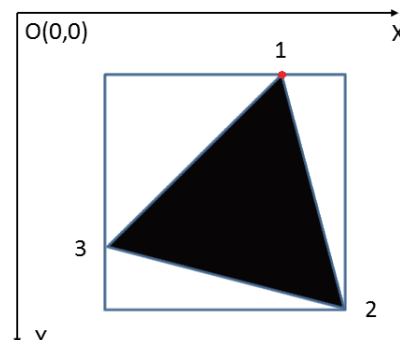

(b)

Fig. 7. (Color online) Determination of the first point for the triangular holes of the first world orientation: (a) left and (b) right image planes. 
holes, as illustrated in Figs. 6(a)-6(c), in the left and right image planes obtained from the double camera. On the left side, the scan intersected the first point at vertex 1, and on the right side, the first point was also vertex 1 . Thus, the first point in this case was vertex 1 .

However, a scanning anomaly was found in the case of the orientation depicted in Fig. 6(d), in which the first points included two different vertices. Figures $8(\mathrm{a})$ and $8(\mathrm{~b})$ show that in the left image plane, the proposed system could identify the first point at vertex 1, but on the right side, the first point was found to be vertex 2. Similarly, Figs. 8(c) and 8(d) show that in the left image plane, the proposed system could identify the first point at vertex 2 , but on the right image plane, the first point is vertex 1 . In this case, the system automatically obtained a point below the original first point (vertex 3) as the first point, because this was the first point scanned that offered congruency between the two image frames.

In the case shown in Fig. 8, Eq. (6) defines the condition necessary to determine the first points of the triangular holes by comparing the image coordinates $1\left(X_{1}, Y_{1}\right)$ and $2\left(X_{2}, Y_{2}\right)$, as follows.

$$
\left|Y_{1}-Y_{2}\right|<e
$$

where $e$ is a threshold that can be set by the user. In this study, $e=10$. If Eq. (6) can be satisfied, then the first point $I\left(X_{F}, Y_{F}\right)$ of a triangular hole will be point $3\left(X_{3}, Y_{3}\right)$ as follows.

$$
\left\{\begin{array}{c}
X_{F}=X_{3} \\
Y_{F}=Y_{3}
\end{array}\right.
$$

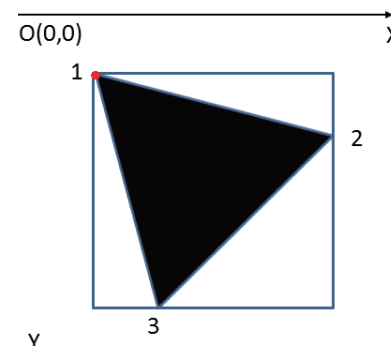

(a)

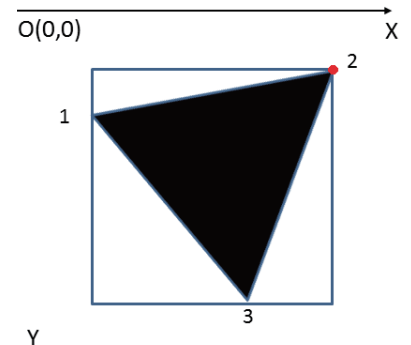

(c)

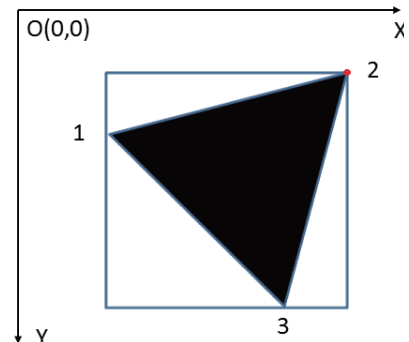

(b)

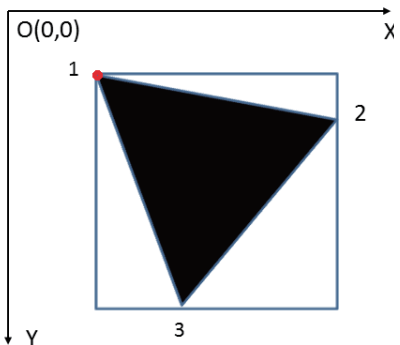

(d)

Fig. 8. (Color online) Determination of the first points for triangular holes of the fourth orientation shown in Fig. 6(d): (a) and (c) left and (b) and (d) right image planes. 
The results of the process for determining the first points of the triangular holes are shown in Fig. 9. Here, the proposed system could identify and mark the positions of the first points of each triangular hole. In Figs. 9(a), 9(c), and 9(d), the positions of the first points are above the horizontal axis, and in Fig. 9(b), the positions of the first points are under the vertical axis of the centers of the triangular holes. The orientation of the triangle in Fig. 9(b) conforms to the examples illustrated in Figs. 6(d) and 8(a)-8(d). The coordinates of these first points and the angular positions of the first point vertices are presented in Table 1.

After the first points were determined, the proposed system identified their coordinates as related to $d x$ and $d y$ in the $X$ - and $Y$-directions, respectively. To determine the orientations of the triangular holes, a reference angle in radians $\left(d_{\text {angle }}\right)$ was calculated as

$$
d_{\text {angle }}=\frac{\left[\left(\operatorname{Atn} \frac{d y}{d x}\right) \times 180\right]}{3.14}
$$

where the Atn function returns the arctangent of a number. The real orientations of the triangular holes can be determined as follows:

- If $d y$ is smaller than 0 , and the absolute value of $d_{\text {angle }}$ is larger than $75^{\circ}$ and smaller than $105^{\circ}$, then the orientation of the triangular hole is $60^{\circ}$.

- If $d y$ is larger than 0 , and the absolute value of $d_{\text {angle }}$ is larger than $75^{\circ}$ and smaller than $105^{\circ}$, then the orientation of the triangular hole is $60^{\circ}$.

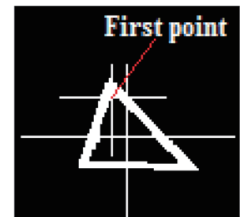

Left image plane

(a)

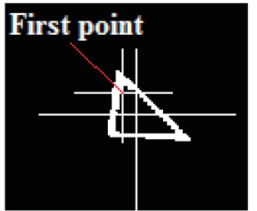

Right image plane

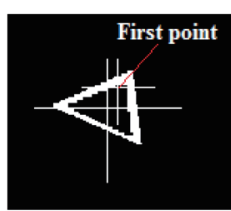

Right image plane

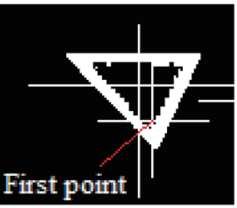

Left image plane

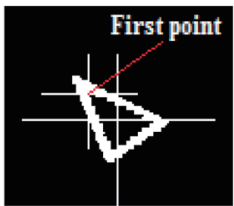

Left image plane

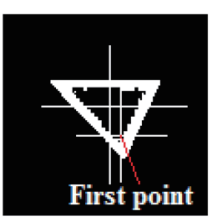

Right image plane

(b)

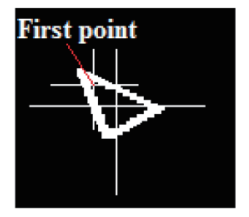

Right image plane

(c)

(d)

Fig. 9. (Color online) Results of the process for determining the first points of the triangular holes: (a) red, (b) green, (c) blue, and (d) yellow triangular holes.

Table 1

World coordinates of the first points of the triangular holes.

\begin{tabular}{lcccc}
\hline Color of & \multicolumn{4}{c}{ World coordinates of first points } \\
\cline { 2 - 5 } triangular hole & $X(\mathrm{~mm})$ & $Y(\mathrm{~mm})$ & $Z(\mathrm{~mm})$ & $\mathrm{A}\left({ }^{\circ}\right)$ \\
\hline Red & 199.162 & 125.963 & 147.212 & 60 \\
Green & 609.981 & 150.618 & 166.356 & 60 \\
Blue & 836.157 & 126.725 & 152.339 & 90 \\
Yellow & 298.444 & 134.092 & 149.823 & 90 \\
\hline
\end{tabular}


- If $d x$ is smaller than 0 , and the absolute value of $d_{\text {angle }}$ is larger than $40^{\circ}$ and smaller than $75^{\circ}$, then the orientation of the triangular hole is $90^{\circ}$.

- If $d x$ is larger than 0 , and the absolute value of $d_{\text {angle }}$ is larger than $40^{\circ}$ and smaller than $75^{\circ}$, then the orientation of the triangular hole is $90^{\circ}$.

The real orientations of the triangular holes are shown in Table 1.

\section{Experimental Procedure}

\subsection{Calibration work}

Camera calibration plays an important role in machine vision systems because it affects the accuracy of the coordinates of the target objects. After completing the camera calibration, we can determine the relationship between the image and world coordinates. Some standard methods for camera calibration were developed in previous studies. ${ }^{(21,22)}$

In this study, we combined these calibration methods to form a coordinate calibration procedure for $2 \mathrm{D}$ and $3 \mathrm{D}$ spaces using the quadratic transformation ${ }^{(23)}$ and the perspective transformation, ${ }^{(5)}$ respectively. This calibration procedure will be described in detail in Sects. 5.1 and 5.2 of this paper.

\subsection{Image processing and object segmentation}

In this section, the classification of the assembly parts and modules is described. As shown in Fig. 10, the assembly modules featured circular, square, and triangular holes, which were color coded red, green, blue, or yellow. The assembly parts are shown in Fig. 11, including spheres, cubes, triangular prisms, and hexagonal prisms, which were red, green, blue, or yellow.

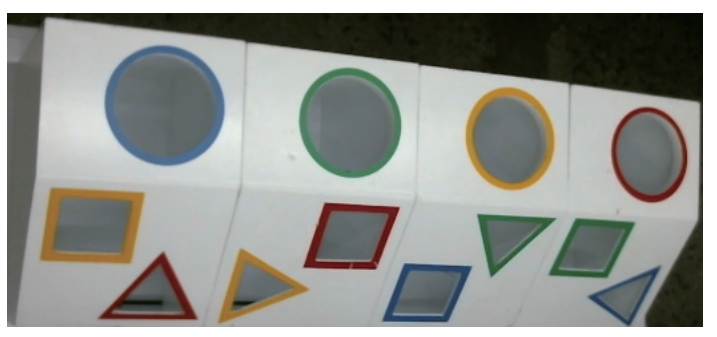

(a)

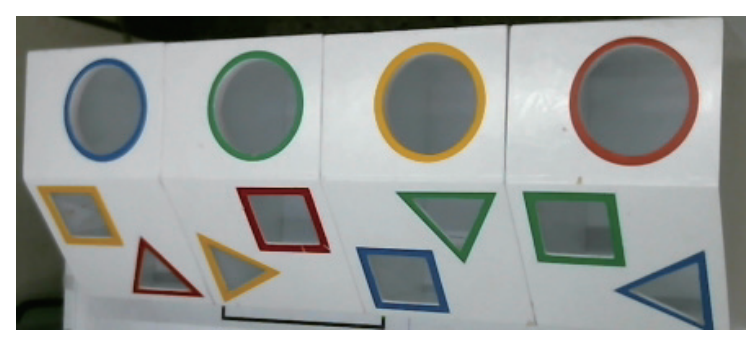

(b)

Fig. 10. (Color online) Color images of the assembly modules for (a) left and (b) right image planes.

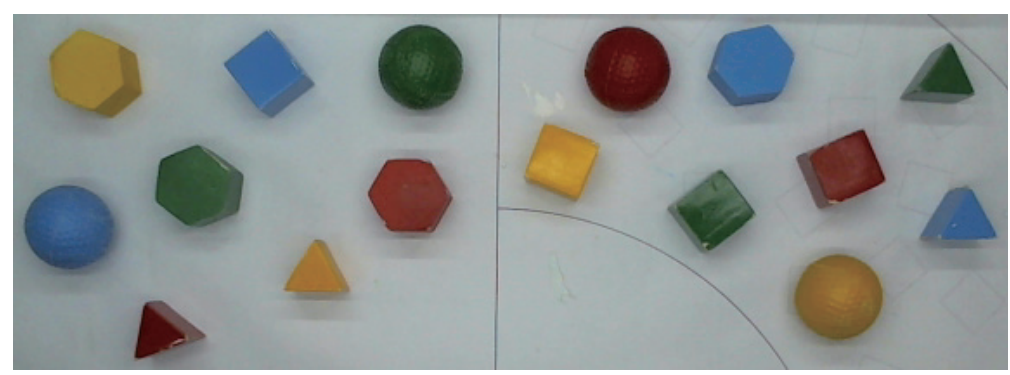

Fig. 11. (Color online) Color images of the assembly parts. 
During the image processing, an additive color model was used to specify the colors. The various color models include the red, green, and blue (RGB) color model, the cyan, magenta, and yellow (CMY) color model, the cyan, magenta, and yellow, key (CMYK) color model, and the hue, saturation, and intensive component (HSI) color model. In this study, the RGB model was used and was based on the Cartesian coordinate system. The components of the color model were specified for each coordinate system and for a subspace within each image system, where each color was represented by a single point.

Using the color subspace of the cube as an example of interest, the RGB values were identified at three corners of the cube, while other colors (cyan, magenta, and yellow) were conscripted at the three other corners. Black was considered as the color of the origin, and white was defined as the opposite color. In captured images, as shown in Figs. 10 and 11, each pixel has three intensities corresponding to red, green, and blue on the RGB model. In a red area, the intensity of the color red is the highest, whereas those of the colors blue and green are lower. In reading a green color, the intensity of green is the highest, while those of red and blue are smaller. In a blue area, the intensity of blue is the highest, and those of red and green are lower. However, in a yellow area, the intensity of blue is normally the lowest, and the intensities of red and green are higher.

To correctly assess the color obtained in the images, a color recognition algorithm was adopted. Here, the values of $R, G$, and $B$ were compared to a threshold using a set of inequalities appropriate for each specified color. For example, Eq. (9) was used to recognize the color red, as follows:

$$
\left\{\begin{array}{c}
R-G>a_{1} \\
R-B>b_{1} \\
G<c_{1} \\
B<d_{1}
\end{array}\right.
$$

where $R, G$, and $B$ are the intensities of red, green, and blue, respectively, at each pixel in the image. All values that satisfy the conditions given in Eq. (9) are set to be 255 (white). If not, they will be set to 0 (black color). Similarly, by comparing the intensity of one color with those of the other colors, green, blue, and yellow can be recognized. The algorithms relative to each color are described in Table 2.

Here, $a_{i}, b_{i}, c_{i}$, and $d_{i}$, are the thresholds of all the colors set in this study $(i=1, \ldots, 4)$. These thresholds, as defined by the user, are shown in Table 3 .

Table 2

Color recognition algorithms.

\begin{tabular}{|c|c|}
\hline $\begin{array}{l}\text { Recognized } \\
\text { color }\end{array}$ & Algorithms \\
\hline Red (R) & $R-G>a_{1} R-B>b_{1} \quad G<c_{1} \quad B<d_{1}$ \\
\hline Green $(\mathrm{G})$ & $G-B>a_{2} G-R>b_{2} \quad B<c_{2} \quad R<d_{2}$ \\
\hline Blue (B) & $B-R>a_{3} B-G>b_{3} \quad R<c_{3} \quad G<d_{3}$ \\
\hline Yellow (Y) & $R-B>a_{4} G-B>b_{4} \quad B<c_{4}$ \\
\hline
\end{tabular}

Table 3

Thresholds used in this study.

\begin{tabular}{lrrrr}
\hline$i$ & \multicolumn{4}{c}{ Thresholds } \\
\cline { 2 - 5 } & $a_{i}$ & $b_{i}$ & $c_{i}$ & \multicolumn{1}{c}{$d_{i}$} \\
\hline 1 & 60 & 60 & 90 & 90 \\
2 & 15 & 15 & 150 & 150 \\
3 & 15 & 15 & 90 & 128 \\
4 & 30 & 30 & 128 & \\
\hline
\end{tabular}


After the binary image was obtained for Blob analysis, the threshold was controlled to obtain the best segments. Then, the image processing began with the extraction of the perimeters of the profiles of the holes or objects. The area was calculated as the number of pixels inside the perimeter. A third measure, compactness, was defined as the ratio of the area to the perimeter. Also, the centroid was calculated as the average of the $x$ - and $y$-coordinates of the pixels inside the perimeter. The centroid was used to obtain the horizontal and vertical axes of the silhouette, which were used to estimate the length and orientation of the object. Finally, the shapes of the objects and the coordinates of the centers were determined, as shown in Figs. 12(a)-12(d) and 13(a)-13(d).

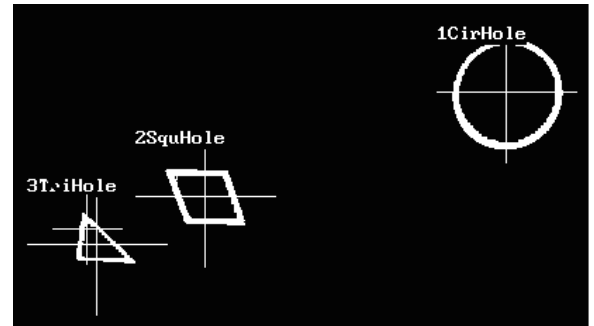

(a)

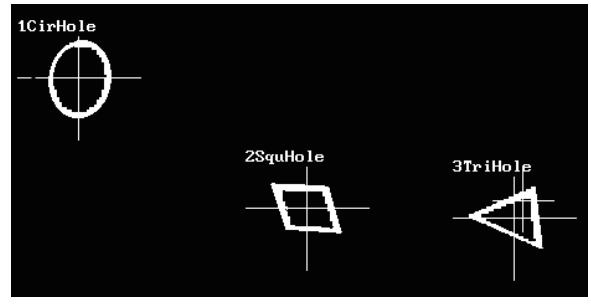

(c)

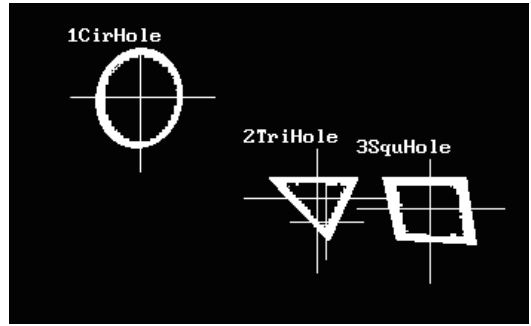

(b)

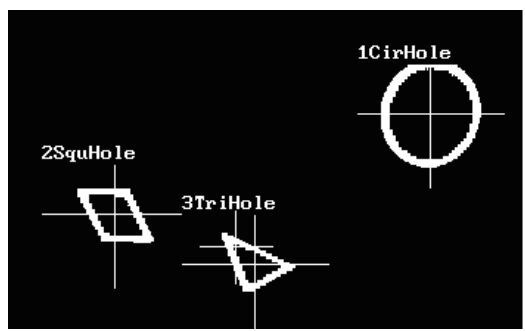

(d)

Fig. 12. Results of the binary right image plane recognition of the holes located on the assembly modules for (a) red, (b) green, (c) blue, and (d) yellow holes.

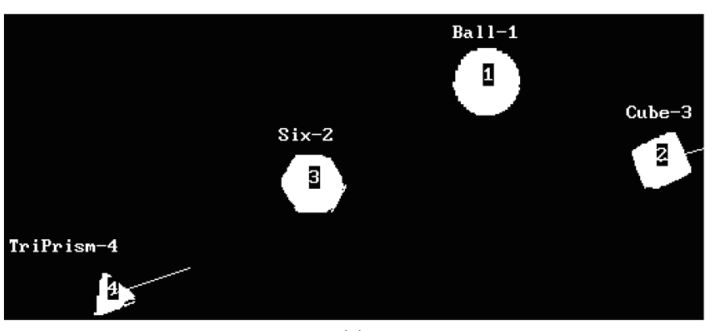

(a)

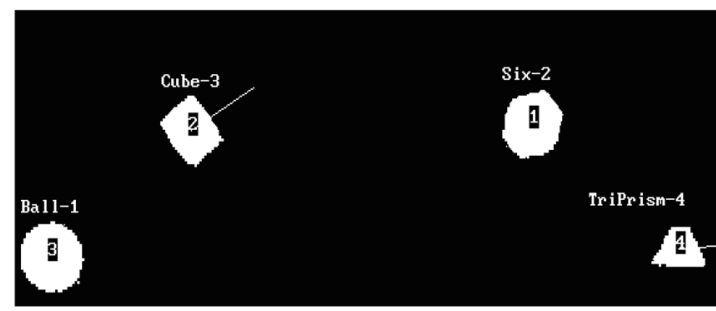

(c)

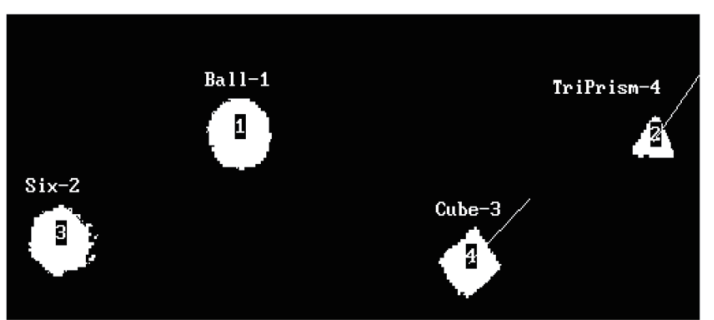

(b)

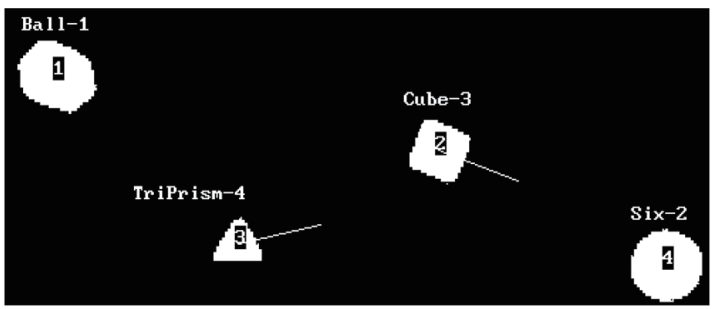

(d)

Fig. 13. Binary image recognition results of the assembly parts for (a) red, (b) green, (c) blue, and (d) yellow shapes. 


\section{Results and Discussion}

\subsection{Calibration and classification results of the assembly parts}

For the 2D coordinate calibration process, a standardized calibration board was used to establish the relationship between the ICS and WCS using the quadratic transformation. The calibration board, as shown in Fig. 14, consists of 60 black circles with a diameter of $17 \mathrm{~mm}$, arranged in 6 rows and 10 columns. The distance between any two black circles is $100 \mathrm{~mm}$ in the $X$-direction and $60 \mathrm{~mm}$ in the $Y$-direction. Among them, the first point was defined as the origin of the world coordinates, which was used as a reference point.

The 2D coordinate calibration pattern of the proposed system shown in Fig. 15 consists of 60 black circles numbered from top to bottom and from left to right. The calibration results shown in Fig. 16 show that the positive maximum deviations are 1.29 and $1.12 \mathrm{~mm}$ in the $X$ - and $Y$-directions, respectively. The negative maximum deviations are -1.50 and $-0.97 \mathrm{~mm}$ in the $X$ and $Y$-directions, respectively.

Concerning the results of the recognition process for the assembly parts, an RGB image of the parts being displayed on the workstation is presented in Fig. 12, while some examples of the recognition results are illustrated in Fig. 13. For each image, the IDs 1, 2, 3, and 4 and the assigned names of the objects are presented. The positions and orientations of all the assembly parts are presented in Table 4.

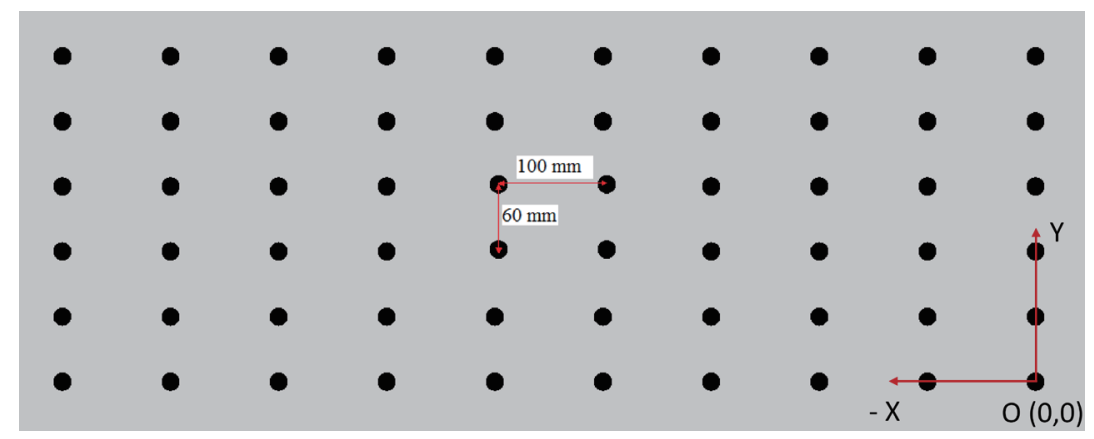

Fig. 14. (Color online) 2D calibration board.

\begin{tabular}{|c|c|c|c|c|c|c|c|}
\hline 政 & 13 & 19 & 25 & 31 & 37 & $\frac{43}{4}$ & $\frac{49}{p}$ \\
\hline 8 外 & 14 & $\frac{2 \theta_{1}}{p}$ & 26 & 32 & 38 & 44 & 50 \\
\hline 种 & 15 & $\frac{21}{p}$ & 27 & $33_{4}$ & 39 & 45. & 51 \\
\hline 10. & 16 & $\frac{22}{p}$ & $\frac{28}{p}$ & 34 & $\frac{4 \sigma_{p}}{p}$ & $\frac{46}{1}$ & 52 \\
\hline 11 & 17 & $\frac{23}{p}$ & $\frac{29}{p}$ & 35 & 41 & 47. & 53 \\
\hline$\frac{12}{4}$ & 18 & 24 & $\frac{30}{1}$ & 36 & 42 & 48 & 54 \\
\hline
\end{tabular}

Fig. 15. Image of 2D calibration board. 


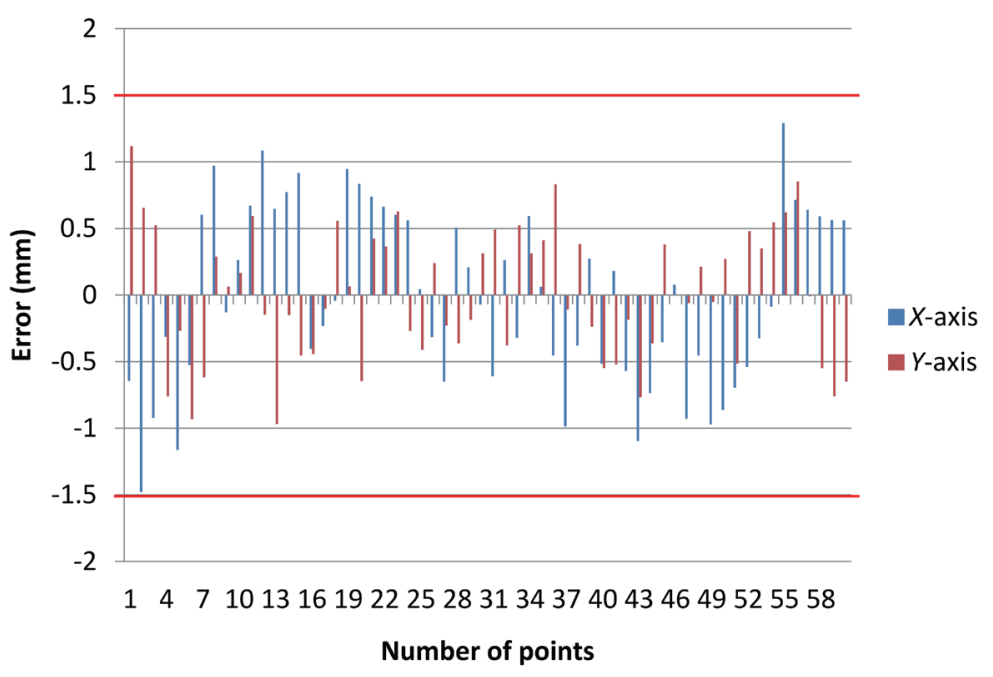

Fig. 16. (Color online) Diagram of 2D calibration error.

Table 4

World coordinates and orientations of assembly parts.

\begin{tabular}{|c|c|c|c|c|c|c|c|c|}
\hline \multirow{2}{*}{ Shape } & \multicolumn{4}{|c|}{ Red } & \multicolumn{4}{|c|}{ Green } \\
\hline & $X(\mathrm{~mm})$ & $Y(\mathrm{~mm})$ & $Z(\mathrm{~mm})$ & $\mathrm{A}\left({ }^{\circ}\right)$ & $X(\mathrm{~mm})$ & $Y(\mathrm{~mm})$ & $Z(\mathrm{~mm})$ & $\mathrm{A}\left({ }^{\circ}\right)$ \\
\hline Cubic & 750.26 & -84.94 & 0 & 23 & 639.28 & -122.53 & 0 & 36 \\
\hline Sphere & 564.59 & -2.68 & 0 & 0 & 389.17 & -2.19 & 0 & 0 \\
\hline Triangular & 162.55 & -229.66 & 0 & 114 & 835.30 & 6.74 & 0 & 25 \\
\hline Hexangular & 379.48 & -111.19 & 0 & 0 & 195.15 & -103.48 & 0 & 0 \\
\hline \multirow{2}{*}{ Shape } & \multicolumn{4}{|c|}{ Blue } & \multicolumn{4}{|c|}{ Yellow } \\
\hline & $X(\mathrm{~mm})$ & $Y(\mathrm{~mm})$ & $Z(\mathrm{~mm})$ & $\mathrm{A}\left({ }^{\circ}\right)$ & $X(\mathrm{~mm})$ & $Y(\mathrm{~mm})$ & $Z(\mathrm{~mm})$ & $\mathrm{A}\left({ }^{\circ}\right)$ \\
\hline Cubic & 258.82 & -8.71 & 0 & 38 & 509.35 & -80.85 & 0 & 69 \\
\hline Sphere & 87.65 & -139.64 & 0 & 0 & 745.76 & -193.65 & 0 & 0 \\
\hline Triangular & 847.25 & -126.61 & 0 & 31 & 301.12 & -176.75 & 0 & 32 \\
\hline Hexangular & 670.18 & 0.9 & 0 & 0 & 109.95 & -7.89 & 0 & 0 \\
\hline
\end{tabular}

\subsection{Calibration and classification results of the assembly modules}

For the 3D coordinate calibration process, a calibration pattern was also used. The calibration pattern, as shown in Fig. 17 included six red squares with a dimension of $30 \mathrm{~mm}$ in both the $X$ - and $Y$-directions. Each point was numbered from the back of the modules to the front and then from left to right using the proposed system. In Fig. 14, the red rectangular center points of the calibration pattern are depicted as points P1 to P6. Of note, the $Z$ dimensions of P3 and P6 of the calibration pattern are shorter to test the variance of height on the basic of the dimensions of the assembly modules. The world coordinates of the centers of all the red squares were measured using the coordinate measurement machine (CMM), as shown in Table 5.

With the 3D coordinate calibration, the forward test was carried out by converting the data from the world coordinates to the image coordinates, and the reverse test was performed from the ICS to the WCS. The results showed that the calibration process yielded correct coordinates for five points (points 1 to 5). However, the forward test for the last point (point 6) had an error 


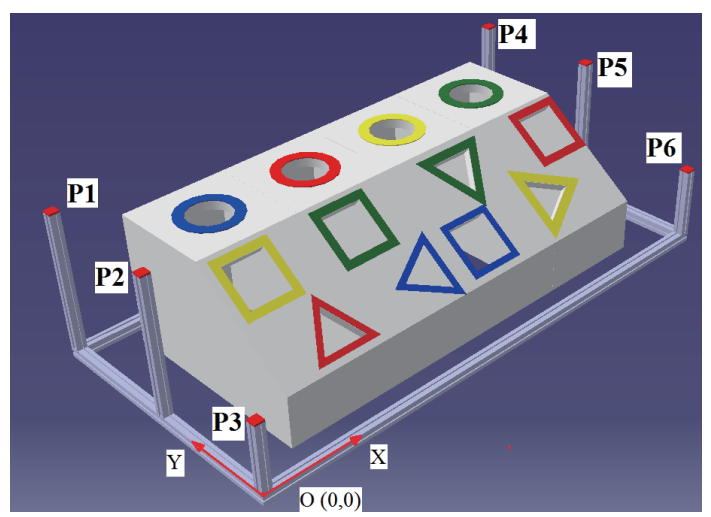

Table 5

World coordinates of the calibration points measured by CMM.

\begin{tabular}{lcrc}
\hline $\begin{array}{l}\text { Calibration } \\
\text { point }\end{array}$ & $X(\mathrm{~mm})$ & $Y(\mathrm{~mm})$ & $Z(\mathrm{~mm})$ \\
\hline P1 & 14.374 & 363.813 & 249.691 \\
P2 & 14.719 & 209.324 & 250.493 \\
P3 & 15.353 & 65.901 & 120.068 \\
P4 & 914.546 & 365.911 & 250.667 \\
P5 & 915.494 & 210.093 & 249.483 \\
P6 & 914.635 & 65.851 & 119.368 \\
\hline
\end{tabular}

Fig. 17. (Color online) 3D calibration pattern.

of 1 pixel. In the reverse test for point 6 (from image coordinate to world coordinate), the world coordinate errors in the $X$-, $Y$-, and $Z$-directions were $0.07,-0.418$ and $-0.063 \mathrm{~mm}$, respectively. Figure 18 depicts the calibration system verification screen. The calibration results are shown in Fig. 19.

Concerning the results of the recognition process for the assembly modules, the RGB images of the modules are shown in Fig. 10, and some examples of the recognition results are illustrated in Fig. 11. For each image, the ID, name, and center lines of the holes are presented. The positions and orientations of all the holes in these assembly modules are presented in Table 6.

\subsection{Implementation}

The robotic arm implemented in this work is depicted in Fig. 20. This robotic arm and its motions are operated within the Cartesian coordinate system, in which the input was defined by the coordinate values $(X, Y, Z, A, B, C)$. Here, $A, B$, and $C$ are the angles of rotation around the $X$-, $Y$-, and $Z$-axes, respectively. Affixed to the robotic arm was an electric gripper, which was used to grasp and manipulate the solid geometric shapes. The electric gripper, a point laser, and two vacuum pads were mounted erect on the $Z$-axis. Two vacuum pads, activated by a pneumatic cylinder, were used to retrieve the triangular, cubic, and hexagonal shapes. These pads could be moved out of the way to prevent them from picking up the balls. The space between the pads was $22 \mathrm{~mm}$, which proved to be ideal for gripping the triangular, cubic, and hexagonal shapes.

In this study, movements were performed point-to-point. The robotic arm was moved to the first reference point of the calibration pattern, as shown in Fig. 9 (not to be confused with the first point of the triangles, as described in Sect. 5.1). The laser pointer then radiated the light used to pinpoint the center of the first circle on the 2D calibration pattern, and then this position was set as a reference point for the robotic arm.

In the execution process, the robotic arm had to perform three steps. In the first step, the robotic arm should pick up all the spheres and hexagonal prisms, and place them into the circular holes according to their respective colors. For the second step, it should pick up the 


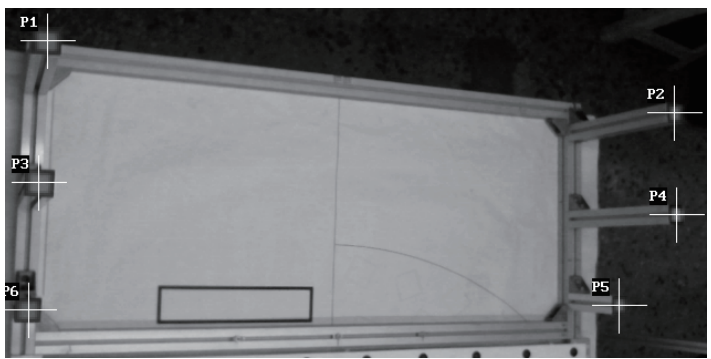

(a)

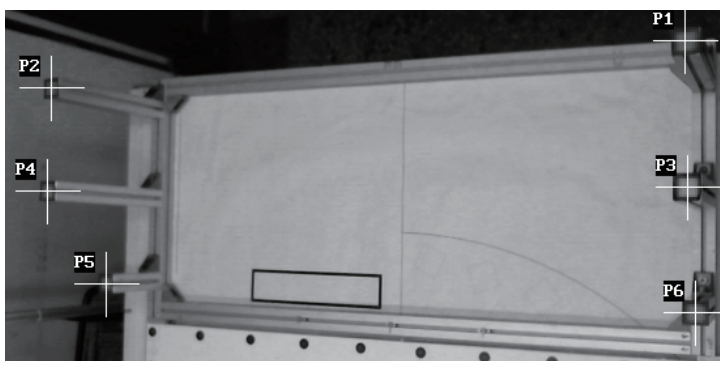

(b)

Fig. 18. Calibration system verification screen for (a) left and (b) right image planes.

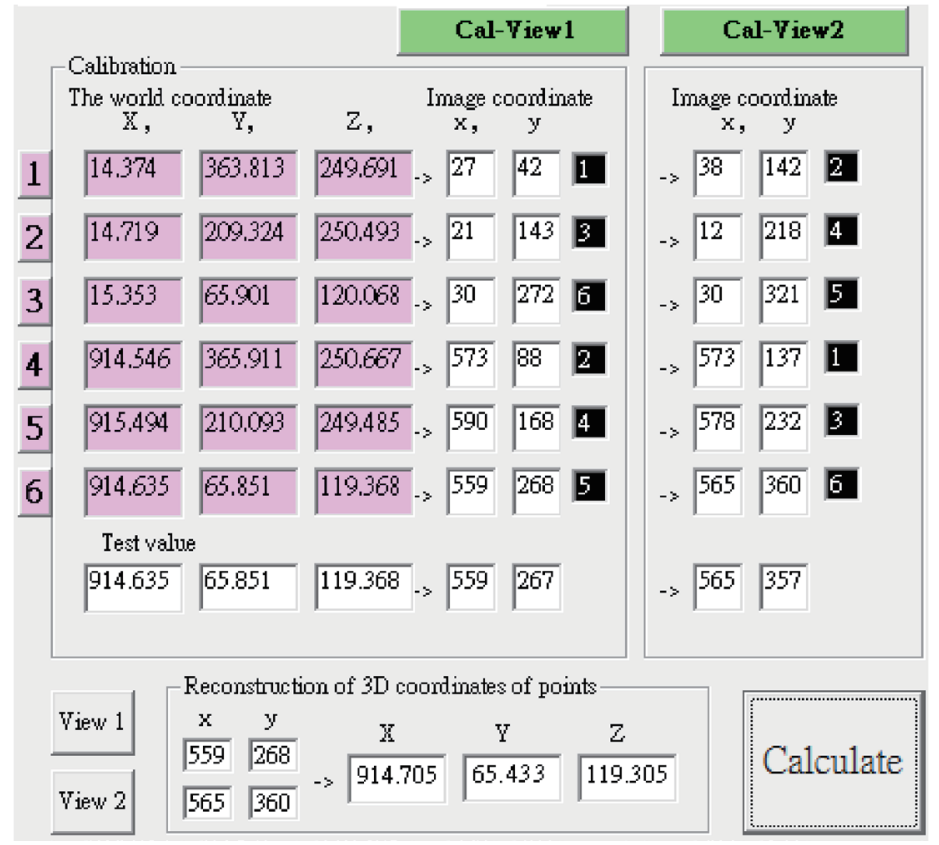

Fig. 19. (Color online) User interface showing the 3D calibration parameters and errors.

Table 6

World coordinates of assembly modules.

\begin{tabular}{|c|c|c|c|c|c|c|c|c|}
\hline \multirow{2}{*}{ Hole shape } & \multicolumn{4}{|c|}{ Red } & \multicolumn{4}{|c|}{ Green } \\
\hline & $X(\mathrm{~mm})$ & $Y(\mathrm{~mm})$ & $Z(\mathrm{~mm})$ & $\mathrm{A}\left({ }^{\circ}\right)$ & $X(\mathrm{~mm})$ & $Y(\mathrm{~mm})$ & $Z(\mathrm{~mm})$ & $\mathrm{A}\left({ }^{\circ}\right)$ \\
\hline Circular & 795.77 & 284.52 & 217.21 & 0 & 187.10 & 294.38 & 218.52 & 0 \\
\hline Square & 407.53 & 169.30 & 181.96 & 0 & 530.36 & 106.41 & 128.61 & 0 \\
\hline Triangular & 201.52 & 99.25 & 126.53 & 60 & 822.63 & 103.49 & 133.51 & 60 \\
\hline \multirow{2}{*}{ Hole shape } & \multicolumn{4}{|c|}{ Blue } & \multicolumn{4}{|c|}{ Yellow } \\
\hline & $X(\mathrm{~mm})$ & $Y(\mathrm{~mm})$ & $Z(\mathrm{~mm})$ & $\mathrm{A}\left({ }^{\circ}\right)$ & $X(\mathrm{~mm})$ & $Y(\mathrm{~mm})$ & $Z(\mathrm{~mm})$ & $\mathrm{A}\left({ }^{\circ}\right)$ \\
\hline Circular & 390.25 & 291.06 & 213.12 & 0 & 593.52 & 288.16 & 216.15 & 0 \\
\hline Square & 733.17 & 164.40 & 179.91 & 0 & 126.91 & 172.62 & 181.31 & 0 \\
\hline Triangular & 607.23 & 176.13 & 188.90 & 90 & 315.77 & 110.81 & 130.24 & 90 \\
\hline
\end{tabular}

cubic shapes, and place them into the square holes according to their respective colors. The last step was to pick up the triangular prisms, and place them in the triangular holes according to their respective colors. 

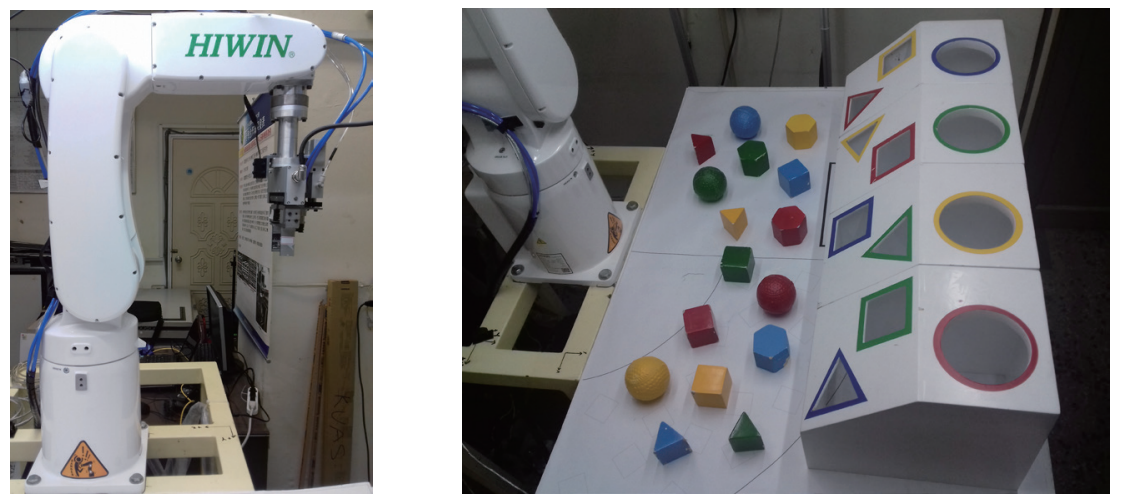

Fig. 20. (Color online) Robotic arm implemented in this work.

In summary, the machine vision system proposed in this work was successfully implemented to ascertain the $2 \mathrm{D}$ and $3 \mathrm{D}$ coordinates of the objects. Then, using the data collected from the visual recognition system, the robotic arm automatically performed the classification and assembly operations.

\section{Conclusions}

In this study, a color object classification and measurement system for a robotic arm was developed using a machine vision system. With this system, the robotic arm could accurately recognize and classify different colored objects. The conclusions drawn from this research are summarized as follows:

- For the 2D object classification and calibration work, the CMOS camera C525 successfully captured the data related to location. The quadratic transformation, which is a plane-toplane transformation, was used to determine the relationship between the image and world coordinates.

- For the 3D object recognition and calibration work, an automatic determination of a position of interest on the calibration pattern was performed successfully. The perspective transformation was used to determine the relationship between the image and world coordinates. - Concerning the image calibration, the conversion process from the WCS to the ICS, as well as the reverse process, of five calibration points (from points 1 to 5) was successful. The error was small and within a suitable accuracy range for the proposed system.

- Algorithms that could ascertain the orientations of different geometries were developed, allowing the robotic arm to position the triangles into their respective holes accordingly.

- By using the RGB color model and choosing suitable thresholds for various colors, including red, blue, green, and yellow, the robotic arm and machine vision system were capable of recognizing and successfully matching each of the parts to its associated hole in the assembly module. The program presented the ID, name, and center lines of the objects and holes accurately. 


\section{References}

1 C. Kavitha and S. D. Ashok: Metrol. Meas. Syst. 24 (2017) 201. https://doi.org/10.1515/mms-2017-0018

2 S. B. Dworkin and T. J. Nye: J. Mater. Process. Technol. 174 (2006) 1. https://doi.org/10.1016/ j.jmatprotec.2004.10.019

3 G. Di Leo, C. Liguori, A. Pietrosanto, and P. Sommella: Opt. Las. Eng. 89 (2017) 162. https://doi.org/10.1016/ j.optlaseng.2016.05.007

4 G. Shuxia, Z. Jiancheng, J. Xiaofeng, P. Yin, and W. Lei: Procedia Eng. 15 (2011) 1807. https://doi.org/10.1016/ j.proeng.2011.08.336

5 N. V. Ngo, Q. C. Hsu, W. L. Hsiao, and C. J. Yang: Adv. Mech. Eng. 9 (2017) 1. https://doi. org $/ 10.1177 / 1687814017717183$

6 R. Xiang, W. He, X. Zhang, D. Wang, and Y. Shan: Measurement 122 (2018) 106. https://doi.org/10.1016/ j.measurement.2018.03.017

7 J. Blasco, N. Aleixos, J. M. Roger, G. Rabatel, and E. Molto: Biosyst. Eng. 83 (2002) 149. https://doi. org/10.1006/bioe.2002.0109

8 P. Nerakae, P. Uangpairoj, and K. Chamniprasart: Procedia Comput. Sci. 96 (2016) 428. https://doi.org/10.1016/ j.procs.2016.08.090

9 B. Iscimen, H. Atasoy, Y. Kutlu, S. Yildirim, and E. Yildirim: Elektron. Elektrotech. 21 (2015) 3. http://dx.doi. org/10.5755/j01.eee.21.6.13749

10 G. Di Fulvio, E. Frontoni, A. Mancini, and P. Zingaretti: Proc. 2014 IEEE/ASME 10th Int. Conf. Mechatronic and Embedded Systems and Applications (MESA, 2014) 1. https://doi.org/10.1109/MESA.2014.6935618

11 N. Rai, B. Rai, and P. Rai: Proc. 2014 2nd Int. Conf. Emerging Technology Trends in Electronics, Communication and Networking (IEEE, 2014) 1. https://doi.org/10.1109/ET2ECN.2014.7044931

12 P. Tsarouchi, G. Michalos, S. Makris, and G. Chryssolouris: Procedia CIRP 9 (2013) 61. https://doi.org/10.1016/ j.procir.2013.06.169

13 X. You and Y. Zheng: Neurocomputing 204 (2016) 222. https://doi.org/10.1016/j.neucom.2015.09.132

14 K. Y. Shin and J. H. Mun: Int. J. Precis. Eng. Manuf. 13 (2012) 283. https://doi.org/10.1007/s12541-012-0035-1

15 L. Deng, G. Lu, Y. Shao, M. Fei, and H. Hu: Neurocomputing 174 (2016) 456. https://doi.org/10.1016/ j.neucom.2015.03.119

16 Y. Ji, A. Yamashita, and H. Asama: Robot. Auton. Syst. 87 (2017) 313. https://doi.org/10.1016/j.robot.2016.09.015

17 Q. Wang, N. Zissler, and R. Holden: Robot. Comput. Integr. Manuf. 29 (2013) 1. https://doi.org/10.1016/ j.rcim.2012.06.003

18 D. Palousek, M. Omasta, D. Koutny, J. Bednar, T. Koutecky, and F. Dokoupil: Opt. Mater. 40 (2015) 1. https:// doi.org/10.1016/j.optmat.2014.11.020

19 W. Zhang, B. Yuchen, and D. Feng: Proc. 2012 24th Chinese Control and Decision Conf. (IEEE, 2012).https:// doi.org/10.1109/CCDC.2012.6244413

20 Q. C. Hsu: Int. J. Mach. Tools Manuf. 43 (2003) 515. https://doi.org/10.1016/S0890-6955(02)00241-9

21 R. Y. Tsai: IEEE J. Robot. Autom. 3 (1987) 323. https://doi.org/10.1109/JRA.1987.1087109

22 Z. Zhang: IEEE Trans. Pattern Anal. Mach. Intell. 22 (2000) 1330. https://doi.org/10.1109/34.888718

23 Q. C. Hsu, N. V. Ngo, and R. H. Ni: Int. J. Adv. Manuf. Technol. 76 (2018) 247. doi: https://doi.org/10.1007/ s00170-018-2888-7

\section{About the Authors}

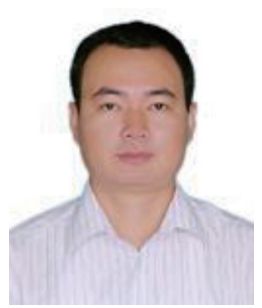

Ngoc-Vu Ngo received his B.S. and M.S. degrees in manufacturing technology from Thai Nguyen University of Technology, Vietnam, in 2004 and 2009, respectively. In 2019, he received his Ph.D. degree in mechanical engineering from the National Kaohsiung University of Science and Technology, Taiwan. At present, he is a lecturer in the Division of Mechatronics, Department of Mechanical Engineering at Thai Nguyen University of Technology, Vietnam. His research interests are in manufacturing technology, computer-aided manufacturing, and machine vision. (ngocvu@tnut.edu.vn) 


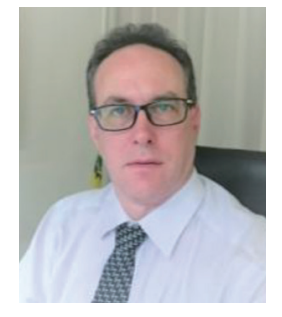

Glen Andrew Porter earned his B.A. degree in physics and mathematics from Carson Newman University, U.S.A., in 1997, and his M.S. in materials science and engineering from the University of Tennessee, U.S.A., in 2000. He received his Ph.D. degree in mechanical engineering from the National Taiwan University of Science and Technology, Taiwan, in 2011. From 2014 to 2019, he served as an assistant professor and a lecturer at the National Kaohsiung University of Science and Technology. His research interests include optoelectronics, optical materials, thin film technology, and manufacturing science. (gaporter1@gmail.com)

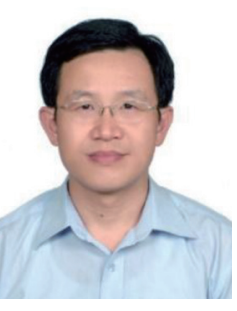

Quang-Cherng Hsu received his B.S., M.S., and Ph.D. degrees from National Cheng Kung University, Taiwan, in 1985, 1987, and 1991, respectively. From 1993 to 1995, he was an engineer at the Metal Industries Research \& Development Centre, Taiwan. From 1995 to 2005, he was an associate professor at the National Kaohsiung University of Applied Sciences, Taiwan. Since 2005, he has been a professor at the National Kaohsiung University of Science and Technology, Taiwan. His research interests are in metal forming, machine vision, and nanoengineering. (hsuqc@nkust.edu.tw) 